\title{
QUEEN'S
UNIVERSITY
BELFAST
}

\section{Laser-Driven Fast Electron Collimation in Targets with Resistivity Boundary}

Ramakrishna, B., Kar, S., Robinson, A. P. L., Adams, D., Markey, K., Quinn, K., Yuan, X. H., McKenna, P., Lancaster, K., Green, J. S., Scott, R. H. H., Norreys, P., Schreiber, J., \& Zepf, M. (2010). Laser-Driven Fast Electron Collimation in Targets with Resistivity Boundary. Physical Review Letters, 105(13), [135001]. https://doi.org/10.1103/PhysRevLett.105.135001

\section{Published in:}

Physical Review Letters

Document Version:

Publisher's PDF, also known as Version of record

Queen's University Belfast - Research Portal:

Link to publication record in Queen's University Belfast Research Portal

Publisher rights

(C) 2010 The American Physical Society

\section{General rights}

Copyright for the publications made accessible via the Queen's University Belfast Research Portal is retained by the author(s) and / or other copyright owners and it is a condition of accessing these publications that users recognise and abide by the legal requirements associated with these rights.

Take down policy

The Research Portal is Queen's institutional repository that provides access to Queen's research output. Every effort has been made to ensure that content in the Research Portal does not infringe any person's rights, or applicable UK laws. If you discover content in the Research Portal that you believe breaches copyright or violates any law, please contact openaccess@qub.ac.uk. 


\title{
Laser-Driven Fast Electron Collimation in Targets with Resistivity Boundary
}

\author{
B. Ramakrishna, ${ }^{1}$ S. Kar, ${ }^{1}$ A. P. L. Robinson, ${ }^{3}$ D. J. Adams, ${ }^{1}$ K. Markey, ${ }^{1}$ M. N. Quinn, ${ }^{2}$ X. H. Yuan, ${ }^{2}$ P. McKenna, ${ }^{2}$ \\ K. L. Lancaster, ${ }^{3}$ J. S. Green, ${ }^{3}$ R. H. H. Scott, ${ }^{3,4}$ P. A. Norreys, ${ }^{3}$ J. Schreiber, ${ }^{4}$ and M. Zepf ${ }^{1}$ \\ ${ }^{1}$ School of Mathematics and Physics, Queen's University of Belfast, Belfast, BT7 1NN, United Kingdom \\ ${ }^{2}$ SUPA, Department of Physics, University of Strathclyde, Glasgow G4 ONG, United Kingdom \\ ${ }^{3}$ Central Laser Facility, Rutherford Appleton Laboratory, Chilton, Oxfordshire, OX11 OQX, United Kingdom \\ ${ }^{4}$ Blackett Laboratory, Imperial College London, Prince Consort Road, London SW7 2BZ, United Kingdom
}

(Received 25 November 2009; published 20 September 2010)

\begin{abstract}
We demonstrate experimentally that the relativistic electron flow in a dense plasma can be efficiently confined and guided in targets exhibiting a high-resistivity-core-low-resistivity-cladding structure analogous to optical waveguides. The relativistic electron beam is shown to be confined to an area of the order of the core diameter $(50 \mu \mathrm{m})$, which has the potential to substantially enhance the coupling efficiency of electrons to the compressed fusion fuel in the Fast Ignitor fusion in full-scale fusion experiments.
\end{abstract}

DOI: 10.1103/PhysRevLett.105.135001

PACS numbers: 52.57.- z, 52.38.Kd

Fast ignition [1] of inertially confined fusion targets has the potential for high-energy gain factors to be achieved with comparably modest total input energy, making this approach attractive as a source of practical fusion energy. For fast ignition to be successful, sufficient energy must be delivered in a short pulse ( $<20$ ps-the ignitor) to a hot spot in the highly compressed $\left(\rho \sim 400 \mathrm{~g} / \mathrm{cm}^{3}\right)$ DT fuel pellet before it decompresses. The most intensely investigated approach is to rely on converting the extremely high power densities that can be achieved with short pulse lasers $\left(>10^{20} \mathrm{~W} \mathrm{~cm}^{-2}[2]\right)$ to relativistic electrons, which in turn heat the fusion fuel on a time scale shorter than the target disassembly time. Understanding the physics of electron production, transport, and deposition in a tightly bounded hot spot are all key to making this approach work with laser parameters close to the theoretical minimum [3].

Initial experiments [4] using a cone insert [5] to facilitate coupling of the short pulse laser to the compressed plasma were highly encouraging. Maintaining these favorable coupling parameters for full-scale fusion pellets brings some additional challenges not encountered by these early experiments. Full-scale experiments will compress the fuel to much a higher density using $100 \times$ more energy in the nanosecond-duration compression beams [6,7]. In such experiments the cone walls will need to be considerably thicker to reduce plasma formation in the cone, and thus a larger stand-off distance will be required. Since the fast electron beam generated by the ignitor laser has a significant angular spread [8], large stand-off distances result in the ignitor energy being coupled into a hot spot much larger than the minimum volume and the energy required for successful ignition and energy gain increases rapidly [3]. Secondly, for divergent electron beam transport, the electron source size-and therefore the laser spot-must be smaller than the desired hot spot diameter $\left(r_{\mathrm{HS}} \rho \sim 0.4 \mathrm{~g} \mathrm{~cm}^{-2}\right.$ or $r_{\mathrm{HS}} \sim 10 \mu \mathrm{m}$ for a typical density of $\rho=400 \mathrm{~g} \mathrm{~cm}^{-3}$ ). Together with the constraint of the maximum pulse duration (typically $r / c_{s} \sim 10 \mathrm{ps} ; r$ is the fuel radius, $c_{s}$ is the speed of sound) and the coupling efficiency this determines the minimum laser intensity for the ignitor pulse (typically exceeding $10^{21} \mathrm{~W} \mathrm{~cm}^{-2}$ ). Assuming the ponderomotive energy scaling for the hot electron temperature as $T_{\text {hot }} \sim\left(I \lambda^{2}\right)^{1 / 2}$ ( $I$ is the intensity and $\lambda$ is the laser wavelength), this minimum intensity would result in electron energies which penetrate more deeply into the fusion fuel than desirable (i.e., electron range $>$ hot spot diameter). This, in turn, would increase the hot spot volume beyond the optimum value and thus increases the required total laser energy to achieve ignition for a fixed laser wavelength $\lambda$. For example, if the electron beam were to heat a hot spot of size $\rho r_{\mathrm{HS}}=1.2 \mathrm{~g} \mathrm{~cm}^{-2}$, the required ignition energy coupled into the hot spot would increase to $55 \mathrm{~kJ}$ from the minimum of $15 \mathrm{~kJ}$. Consequently, developing a technique that allows the electron beam to be focused adds additional control and relaxes the constraints.

Here we present the first experimental investigation that demonstrates that targets with suitably shaped boundaries in their material composition can collimate the electron flow over large lengths in two dimensions without significant loss in transport efficiency. This opens up the way to targets that enable the electron source size to be controlled independently of the laser intensity for fixed laser parameters. For example, this could allow electrons from a large source area to efficiently reach the hot spot. Furthermore it enables significant stand-off distances between relativistic electron source and fuel pellet without a substantial drop in coupling efficiency due to geometric spreading and hence represents a major advance for the Fast Ignitor.

The effect of material boundaries with strongly varying resistivity on magnetic field generation and hence relativistic electron flow is described in detail in the theoretical work of Robinson and Sherlock [9] and our recent proof of the principle experiment [10]. Briefly, the laser interaction 
with an overdense plasma produces a divergent relativistic electron beam at the critical density surface of the plasma $[8,11]$. The large instantaneous currents $\left(10^{6}-10^{9} \mathrm{~A}\right)$ lead to strong collective effects [12-14] which affect the overall divergence and fine structure (i.e., filamentation [15]) of the beam. Robinson and Sherlock highlighted the potential for harnessing these self-generated fields for guided electron beam transport in targets specifically designed to give rapid magnetic field growth at a well-defined boundary. The magnetic field growth can be derived from Faraday's law and Ohm's law as

$$
\frac{\partial \vec{B}}{\partial t}=\eta \vec{\nabla} \times \vec{j}+(\vec{\nabla} \eta) \times \vec{j},
$$

where $\eta$ is the resistivity and $\vec{j}$ is the fast electron current density. The first term corresponds to magnetic fields that act to push fast electrons towards regions of higher fast electron density, while the second term pushes the fast electrons towards regions of higher resistivity. This implies that a target with a sharp radial resistivity boundary should build up strong magnetic fields (many megagauss) at the boundary between a high-resistivity "core" and lowresistivity "cladding" which act to collimate the flow of fast electrons [9]. Here we used the $Z$ dependence of the resistivity [e.g., $\eta=10^{-4} Z \ln (\Lambda) T^{-3 / 2}$ in the Spitzer regime; $Z$ is the nuclear charge, $\ln (\Lambda)$ is the Coulomb logarithm, and $T$ is the temperature] to achieve this aim. The guiding targets were engineered in such a way that the core has a higher resistivity than the cladding. The materials chosen for this experiment were 25 or $50 \mu \mathrm{m}$ Fe wires surrounded by aluminum to make up a total target radius of $250 \mu \mathrm{m}$ [Fig. 1]. Important factors in the choice of material configuration are manufacturing issues and using a high $Z$ material that also has a higher cold resistivity than the low $Z$ material. This ensures that the resistivity gradient retains the correct sign across the full range of target temperatures [9]. The combination of Fe-Al fulfills these requirements with a room temperature resistivity ratio of 3.7 and a $Z$ ratio of 2 and should, therefore, lead to effective magnetic field generation and electron guiding.

The target design was verified as using the 3D ZEPHYROS hybrid code, which treats fast electrons using the VlasovFokker-Planck approach [16] with background electrons included similarly to the code of Davies [17]. The a) Laser, $150 \mathrm{~J},<.8 \mathrm{ps}$

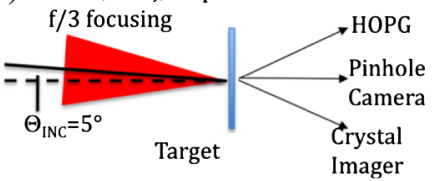

b)

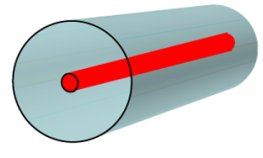

FIG. 1 (color online). (a) Schematic of experimental layout. Laser $(\lambda=1053 \mathrm{~nm})$ is $f / 3$ focused under an angle of $5^{\circ}$ on the target with up to $150 \mathrm{~J}, 0.8 \mathrm{ps}$, and peak intensities $\sim 10^{20} \mathrm{~W} \mathrm{~cm}^{-2}$. (b) Guiding target showing Fe core [dark shading (red), 25 or $50 \mu \mathrm{m}$ diameter] and Al cladding $(250 \mu \mathrm{m}$ diameter). The laser is incident on the axis of the Fe core. simulations were carried out in a $200 \times 200 \times 200 \mu \mathrm{m}$ box with the $1 \mu \mathrm{m}$ cubic cells. The transverse absorption profile is determined by $I=\beta I_{0} \cos ^{2}\left(\pi r / 2 r_{\text {spot }}\right)$, where $\beta$ is the laser to fast electron conversion efficiency, $I_{0}$ is the laser intensity, and $r_{\text {spot }}$ is the FWHM of laser focal spot. In the simulations, $\beta$ was set to $0.3, I_{0}$ was set to $10^{20} \mathrm{~W} \mathrm{~cm}^{-2}$, and $r_{\text {spot }}$ was set to $20 \mu \mathrm{m}$ to match the experiment. The electrons were injected with an exponential distribution $f(E)=\exp (-E /\langle E\rangle) /\langle E\rangle(\langle E\rangle$ is the average fast electron energy) over a solid angle subtended by $2 \theta_{\text {div }}=60^{\circ}$. The simulations used a $500 \mathrm{fs}$ pulse and were run up to 1.5 ps. Figure 2 shows the simulation results for three fast electron temperatures $(9,3$, and $1 \mathrm{MeV})$ which were used to determine the robustness of the scheme. In all cases effective collimation was observed due to the rapid magnetic field growth at the resistivity boundaries demonstrating the robustness of the scheme. This is in contrast with the divergent transport with angles close to initial divergence which is typical of experiments and simulations performed with plain foil targets. The simulations also shed light on the dynamics of the collimation process. At early times the effect of the resistivity gradient (the $\vec{\nabla} \eta \times \vec{j}$ term) dominates. At later times the field growth due to the current density gradient $(\eta \vec{\nabla} \times \vec{j})$ is the leading contribution [9]. Consequently the collimating field is anticipated to remain stable for the much longer pulses required for full-scale ignition experiments.

The experiment was performed at Rutherford Appleton Laboratory employing the VULCAN Petawatt laser system. After reflection from a plasma mirror [18], the laser delivered $150 \mathrm{~J}$ of energy on target in $\sim 0.8 \mathrm{ps}$. The laser was focused to a peak intensity of $\sim 10^{20} \mathrm{~W} / \mathrm{cm}^{2}$ on target at a $5^{\circ}$ angle of incidence [Fig. 1]. The primary diagnostic of the electron transport was $\mathrm{Cu} K \alpha$ radiation emitted from a $15 \mu \mathrm{m} \mathrm{Cu}$ tracer layer at the rear side of all the targets. The size of the emitting region was diagnosed using an $\mathrm{x}$-ray pinhole camera (with resolution set by $50 \mu \mathrm{m}$ pinhole
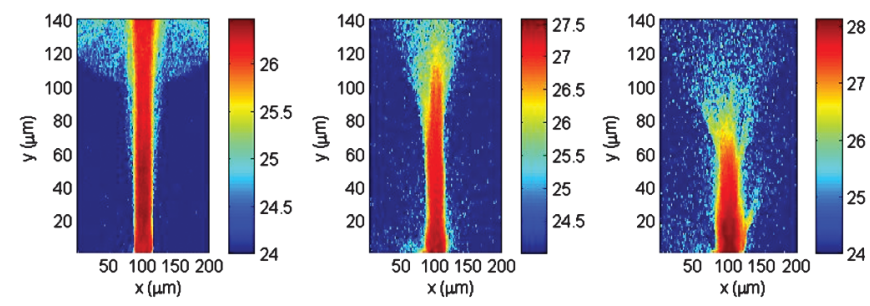

FIG. 2 (color). Fast electron density distribution (on logarithmic scale, top) at 1.5 ps obtained from ZEPHYROS code for Fe-Al guiding targets with $25 \mu \mathrm{m} \mathrm{Fe}$ core for temperatures of $9 \mathrm{MeV}$ (left panel), $3 \mathrm{MeV}$ (center panel) and $1 \mathrm{MeV}$ (right panel). Color bar is in units of $\log \left(N_{e}\left[\mathrm{~m}^{-3}\right]\right)$. All three cases show clear confinement of the electron beam to the Fe core despite electron being injected with a full cone angle of $60^{\circ}$. The confinement is clearly robust with respect to variations in hot electron temperature. Note that the range of the electrons is substantially reduced compared to single particle ranges by collective stopping ( $\sim 80 \mu \mathrm{m}$ rather than $\sim 400 \mu \mathrm{m}$ for $1 \mathrm{MeV}$ electrons). 
diameter), spherical quartz crystal imager ( $8 \mu \mathrm{m}$ resolution), and via source broadening using a highly ordered pyrolytic graphite (HOPG) spectrometer $(\sim 30 \mu \mathrm{m}$ resolution). The imaging crystal did not provide enough signal for thick targets $(>100 \mu \mathrm{m} \mathrm{Fe}$ core length) where the collimation effect is the clearest. The pinhole camera had four $50 \mu \mathrm{m}$ pinholes which were filtered to select different spectral ranges in the range of 1.7 to $>10 \mathrm{keV}$ and an image plate as a detector. The HOPG crystal provided data on the relative $K \alpha$ yield of the targets as well as an independent measure of source size due to a spectral linewidth dominated by source broadening (source broadened widths of $60-80 \mathrm{eV}$ were observed, while typical spectral widths are $\sim 20 \mathrm{eV}$ for comparable targets [19]). HOPG is particularly suited for this experiment, because the unique crystal plane structure results in a combination of highly efficient x-ray diffraction, and mosaic parafocusing $[20,21]$. The agreement between source sizes inferred from the HOPG, pinhole, and crystal imagers was very good [Fig. 3]. The agreement to within experimental error demonstrates that HOPG source broadening is indeed a good way to measure the x-ray source size and that source broadening dominates the measured linewidth under our conditions. Raw data from the crystal imager can be seen in Fig. 4. The measured source size of $\sim 68 \mu \mathrm{m}$ is much larger than the resolution of the crystal imager and demonstrates that the lower resolution of HOPG and pinhole camera had no significant impact on the obtained results.
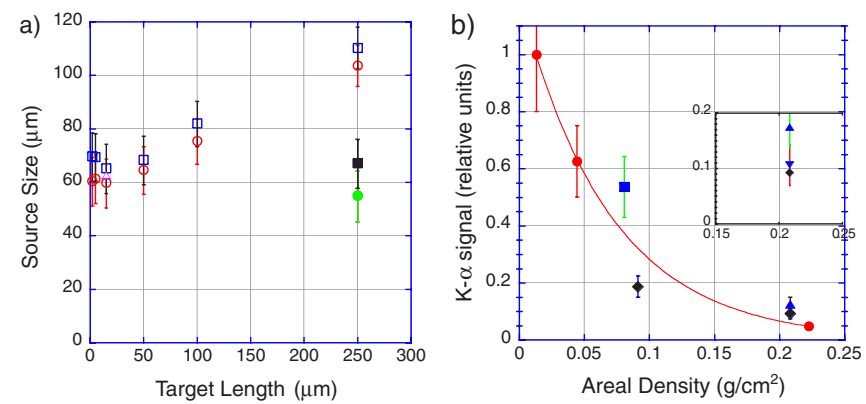

FIG. 3 (color online). FWHM x-ray spot size (a) for uniform targets (open symbols) and guiding targets (filled symbols). Measurements with the crystal imager are shown as triangles, $\mathrm{x}$-ray pinhole camera as circles, and HOPG data as squares. The effect of collimation is clearly visible at $250 \mu \mathrm{m}$ target thickness resulting in near resolution limited source sizes. The data points represent an average over the data set for each thickness for clarity with the collimation data set taken over four shots. The uncertainties are calculated based on the measurement error in the $\mathrm{x}$-ray spot size. (b) $K \alpha$ yield from HOPG spectrometer for $\mathrm{Cu}$ (circles, multishot average), and $\mathrm{Al}$ (squares) and $\mathrm{Fe}$ foils (diamonds), guiding targets (triangle, multishot average). Fe and Al foils had $15 \mu \mathrm{m} \mathrm{Cu}$ backing at the rear. The data for $>0.013 \mathrm{~g} \mathrm{~cm}^{-2}(15 \mu \mathrm{m} \mathrm{Cu})$ shows an exponential decay with areal density. A comparison between Fe reference target (diamond) and guiding targets (25 $\mu \mathrm{m}$ diameter, up triangle; $50 \mu \mathrm{m}$ down triangle) is shown in an enlarged inset for clarity. The error bars represent the level of shot-to-shot fluctuation observed in the experiment.
The effectiveness of the guiding targets was investigated by comparing plain foils of a given thickness with guiding targets of the same thickness (e.g., reference foil thickness $=$ core length; reference foils were $5 \times 5 \mathrm{~mm}^{2}$ ). Figure 3 shows a comparison between multishot averages for targets of different thicknesses. For plain foil targets (the reference case) the divergent nature of the electron beam leads to an increase in the size of the emission region with increasing target thickness (increasing from $60 \mu \mathrm{m}$ diameter for $15 \mu \mathrm{m}$ thick foils to around $110 \mu \mathrm{m}$ diameter for the thickest foils). For guiding targets, effective collimation should lead to a source size comparable to that for the thinnest targets. In agreement with this expectation, the guiding targets with $250 \mu \mathrm{m}$ thickness do indeed exhibit source sizes around $60 \mu \mathrm{m}$ diameter comparable to targets of $<15 \mu \mathrm{m}$ thickness. Figure 4 shows a comparison of the pinhole image filtered to between a reference $\mathrm{Fe}$ foil and guiding target. The reduction in source size is clearly visible. Also visible is the significant reduction in signal outside the main spot, demonstrating that the guiding target is effective at suppressing the global beam spread and also effectively suppresses the diffuse halo caused by small angle scattering.

The overall divergence calculated back to a point source at the foil surface is about $27^{\circ}$ for $250 \mu \mathrm{m}$ thick targets. Note, that for thin foils, the observed size of the $K \alpha$ emission region is not determined by the fast electron source size but by transport effects (primarily refluxing

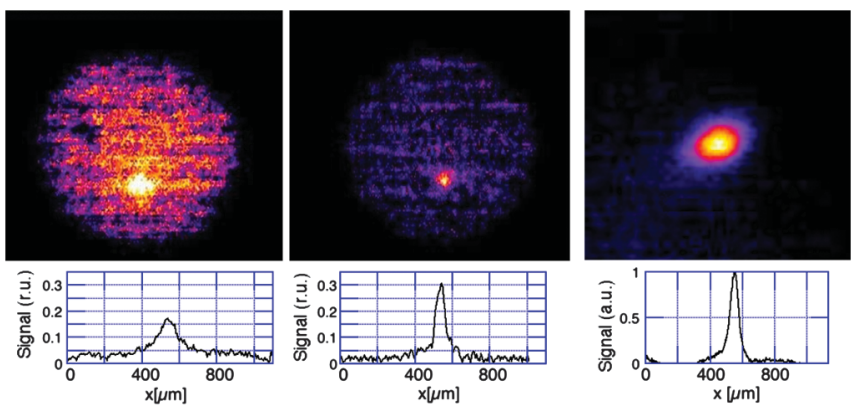

FIG. 4 (color). X-ray emission images of the target rear surface and lineouts showing the reduction of the emission spot size. Comparison of the pinhole camera image of the reference target $(250 \mu \mathrm{m} \mathrm{Fe}$ foil, left) and the guiding target $(250 \mu \mathrm{m}$ long; $50 \mu \mathrm{m}$ diameter $\mathrm{Fe}$ core in $\mathrm{Al}$, center) shows a significantly smaller $\mathrm{x}$-ray spot size for the guiding target. The reduction in FWHM is from $\sim 110 \mu \mathrm{m}$ to $\sim 60 \mu \mathrm{m}$ and the emission far from the spot is also significantly reduced. Both images were filtered for $\mathrm{Cu} K \alpha$ emission using $10 \mu \mathrm{m} \mathrm{Ni}$ and $10 \mu \mathrm{m}$ Ti foils. Guiding targets result in more intense $\mathrm{x}$-ray radiation as shown by the lineouts. The intensity ratio after correction for the finite resolution due to the $50 \mu \mathrm{m}$ pinhole and viewing angle is 3.1:1, close to the value of 3.2-3.3 expected from the source size measurements. For comparison, an image of a $15 \mu \mathrm{m}$ Copper foil taken with the crystal imager with $8 \mu \mathrm{m}$ spatial resolution (right). The size of the $K \alpha$ emission region in this case is again $\sim 60 \mu \mathrm{m}$ on all diagnostics, demonstrating that the observed emission spot size is not limited by the resolution of the pinhole camera (lineout normalized to peak intensity). 
in our case) [22]. The smallest sources observed for thin foils are around 50-60 $\mu \mathrm{m}$ on all three diagnostics (compared to the few $\mu \mathrm{m}$ resolution of the imaging crystal). Overall the observed source sizes and divergence are within the variation reported in previously published data where the electron beam divergence has been measured using high $Z$ buried layer targets in targets with $10 \mathrm{~s}$ of $\mu \mathrm{m}$ thickness [23-26]. The reported angles vary from $20^{\circ}-30^{\circ}$ [23] to $40^{\circ}$ [27]. Note that while the paper by Lancaster et al. [8] reports a larger divergence angle of more than $50^{\circ}$, this was measured over quite short target lengths $(15-60 \mu \mathrm{m})$ and is thus subject to significant uncertainty. Indeed our data-while inferring a smaller divergence angle for the reference foil-is still consistent with Lancaster et al. to within the uncertainty of both sets of measurements (60 $\mu \mathrm{m} \pm 8 \mu \mathrm{m}$ in [6] compared to our measurement of $65 \mu \mathrm{m} \pm 10 \mu \mathrm{m}$ for $50 \mu \mathrm{m}$ thick targets).

Figure 3(b) shows the variation of $K \alpha$ photon yield on the target areal density. The result for plain $\mathrm{Cu}$ foils shows the expected exponential decay [28]. The rate of decay of the signal in $\mathrm{Cu}$ targets indicates an effective range of $\sim 100 \mathrm{mg} / \mathrm{cm}^{2}$, consistent with recent measurements using cone and wire targets [29]. Our simulations show that the range of hot electrons is substantially reduced compared to the single particle range [Fig. 2] by the electrostatic field required to drive the return current [19] and that the observed range depends on electron distribution function and absorption fraction, making precise estimates of the electron temperature difficult. However, it is clear from the simulations shown in Fig. 2 that the results are consistent with hot electron temperature below that predicted by the ponderomotive scaling [30]. This is consistent with recent studies suggesting reduced hot electron temperature interactions with a steep density gradient [31], which are expected for our experimental parameters due to the use of a plasma mirror combined with ponderomotive steepening [32]. A direct comparison of the $K \alpha$ yield obtained for $250 \mu \mathrm{m} \mathrm{Fe}$ reference targets and guided targets shows that to within the shot-to-shot fluctuations, the electrons are confined to the Fe core [Fig. 3(b)]. Indeed the yield on the guiding shots is slightly higher than on the reference shot, suggesting that shot-to-shot fluctuations are very significant. Given the significant experimental uncertainty, a lower bound for the trapping efficiency (defined as the ratio between reference and guiding target $K \alpha$ signal) over $250 \mu \mathrm{m}$ of $\sim 70 \%$ can be estimated. In full-scale ignition experiments the total transport distance will generally be substantially shorter than in our experiment and current designs have wall thicknesses of $50 \mu \mathrm{m}$ or less. Hence one would anticipate higher guiding fractions for these shorter guides, since most guiding losses are likely to occur at large depths where the electron beam has been strongly attenuated and the collimating magnetic field is lower.
In conclusion, we have demonstrated for the first time that targets with a resistivity boundary can efficiently collimate and confine relativistic electron beams over large distances into a narrow cylinder. Simulations show that the fast electrons are confined by the self-generated azimuthal magnetic field and guided in the high-resistivity core. Such targets allow substantially higher design flexibility for the fast ignitor and may have a substantial impact on ignition facility design.

The authors acknowledge support from R. A.L. staff, especially in target manufacture, from EPSRC (Grant No. EP/E048668/1) and M. Borghesi for helpful discussions. M. Z. acknowledges support from the Royal Society.

[1] M. Tabak et al., Phys. Plasmas 1, 1626 (1994).

[2] C. N. Danson et al., Nucl. Fusion 44, S239 (2004).

[3] S. Atzeni et al., Phys. Plasmas 6, 3316 (1999).

[4] R. Kodama et al., Nature (London) 412, 798 (2001).

[5] P. Norreys et al., Phys. Plasmas 7, 3721 (2000).

[6] J. Lindl et al., Phys. Plasmas 11, 339 (2004).

[7] J. Lindl, Phys. Plasmas 2, 3933 (1995).

[8] K. L. Lancaster et al., Phys. Rev. Lett. 98, 125002 (2007); J. Green et al., Phys. Rev. Lett. 100, 015003 (2008).

[9] A. P. L. Robinson and M. Sherlock, Phys. Plasmas 14, 083105 (2007).

[10] S Kar et al., Phys. Rev. Lett. 102, 055001 (2009).

[11] M. Santala et al., Phys. Rev. Lett. 84, 1459 (2000).

[12] A. R. Bell et al., Plasma Phys. Controlled Fusion 39, 653 (1997).

[13] J. R. Davies et al., Phys. Rev. E 56, 7193 (1997).

[14] A. R. Bell and R. J. Kingham, Phys. Rev. Lett. 91, 035003 (2003).

[15] Y. Sentoku et al., Phys. Plasmas 7, 689 (2000).

[16] A. R. Bell and R. J. Kingham, Phys. Rev. Lett. 91, 035003 (2003).

[17] J. R. Davies, Phys. Rev. E 65, 026407 (2002).

[18] B. Dromey et al., Rev. Sci. Instrum. 75, 645 (2004).

[19] G. Gregori et al., Contrib. Plasma Phys. 45, 284 (2005).

[20] A. W. Moore, Highly Oriented Pyrolytic Graphite, Chemistry And Physics Of Carbon (Marcel Dekker, New York, 1973), Vol. 11.

[21] G. Gregori et al., Rev. Sci. Instrum. 75, 3747 (2004).

[22] A. J. MacKinnon, Phys. Rev. Lett. 88, 215006 (2002).

[23] R. Kodama, P. A. Norreys, K. Mima et al., Nature (London) 432, 1005 (2004).

[24] M. Tatarakis et al., Phys. Rev. Lett. 81, 999 (1998).

[25] M. Borghesi et al., Phys. Rev. Lett. 83, 4309 (1999).

[26] L. Gremillet et al., Phys. Rev. Lett. 83, 5015 (1999).

[27] R. B. Stephens et al., Phys. Rev. E 69, 066414 (2004).

[28] D. Salzmann et al., Phys. Rev. E 65, 036402 (2002).

[29] J. A. King et al., Phys. Plasmas 16, 020701 (2009).

[30] S. C. Wilks et al., Phys. Rev. Lett. 69, 1383 (1992).

[31] A. Kemp, Y. Sentoku, and M. Tabak, Phys. Rev. Lett. 101, 075004 (2008).

[32] B. Dromey et al., Phys. Rev. Lett. 99, 085001 (2007). 